$\begin{array}{lcc}\text { Tükiye Tarımsal Araştırmalar Dergisi } & \text { Turk J Agric Res } \\ \text { dergipark.gov.tr/tutad } & \text { 2018, 5(2): 120-124 } & \text { TÜTAD } \\ \text { ISSN: 2148-2306 } & \text { e-ISSN: 2528-858X } \\ \text { doi: } 10.19159 / \text { tutad.369079 }\end{array}$

\title{
Studies on the Fauna of Papilionoidea and Hesperioidea (Lepidoptera) in Erbil Province (Northern Iraq)*
}

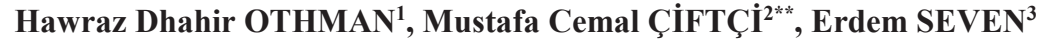 \\ ${ }^{1}$ Ministry of Education, Diroctarite of Koya Education, Hewa High School for Boys, Erbil, IRAQ \\ ${ }^{2}$ Siirt University, Faculty of Agriculture, Department of Plant Protection, Siirt, TURKEY \\ ${ }^{3}$ Batman University, School of Tourism and Hotel Management, Department of Gastronomy and Culinary Arts, Batman, TURKEY
}

\begin{tabular}{|c|c|}
\hline Received: 20.12 .2017 & Accepted: 02.05 .2018 \\
\hline \multicolumn{2}{|l|}{ ORCID ID (By author order) } \\
\hline \multicolumn{2}{|c|}{ (1D)orcid.org/0000-0002-8248-068X (D)orcid.org/0000-0003-2386-6969 (D)orcid.org/0000-0002-7587-5341 } \\
\hline
\end{tabular}

Abstract: In this study, the butterfly fauna of Erbil Province (northern region of Iraq) is investigated for the first time. For this purpose, 1240 collected specimens are evaluated and 55 species are recorded belonging to superfamilies Papilionoidea and Hesperioidea. Valid scientific names, materials, collecting localities and distributions of each species in the region, are given.

Keywords: Butterflies, Papilionoidea, Hesperioidea, fauna, Erbil, Iraq

\section{Introduction}

The oldest literature dealing with the butterflies of Iraq goes back to the beginning of the 20th century. First comprehensive studies about butterflies in the northern region of Iraq were published by Wiltshire (1937a, 1937b, 1939a, 1939b, 1957) and Higgins (1958) with some papers. Koçak and Kemal (2001) presented 145 butterfly species from Iraq and same authors increased this number to 151 species from Iraq (Kemal and Koçak, 2011). Also, Lahony et al. (2013) and Tshikolovets et al. (2014) have given some butterfly species in their studies about the biodiversity of northern Iraq. And, studies on the butterflies of nearby areas were published by: Graves (1925); Hemming (1932); Larsen (1974); Hardy (1995); Kemal and Koçak (2011); Koçak and Kemal (2007); Akın (2008); Seven (2010); Koçak et al. (2011); Koçak and Kemal (2014, 2015a, 2015b) and Abusarhan et al. (2016).

This research is the first study on the butterfly species of Erbil Province. This study aimed to determine the Papilionoidea and Hesperioidea (Lepidoptera) fauna of Erbil province.

\section{Materials and Methods}

In this study, 1240 specimens of butterflies were collected between April-June 2016 and MarchOctober 2017, from Erbil Province in the north of Iraq. They were caught using a long-handled butterfly sweep net. After being captured, specimens were killed with ethyl acetate and packed. Adults were prepared according to standard entomological procedures. The materials are deposited in the Entomology Laboratory of Department of Biology in Siirt University.

Erbil Province is the capital city of the northern region of Iraq, which borders Turkey and Iran in the north and north-east respectively; it also borders the provinces of Sulaymaniyah and Kirkuk on the east, south-east and south (Figure 1). The province is far from the water bodies, there are the great Zab River and the small Zab River passing through the city's outskirts.

The following sources have been used in the taxonomy, distribution, fauna, and identification of the species: Wiltshire (1937a, 1937b, 1939a, 1939b, 1957), Higgins (1958), Aussem (1980), Geiger et al. 


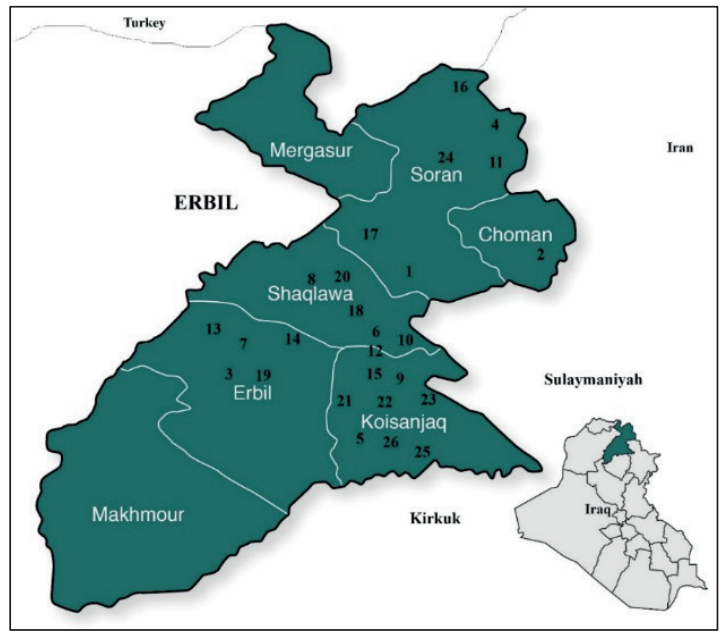

Figure 1. Location of Erbil Province in Iraq [Modified from Anonymous (2017)]
(1988), Seyer (1988), Coenen and Olivier (1989), Hesselbarth et al. (1995), Koçak and Kemal (2001), Freina and De Naderi (2003), Benyamini et al. (2007), Kemal and Koçak (2011), Back (2012), Lahony et al. (2013) and Tshikolovets et al. (2014).

Dates of studies, coordinates and altitudes are presented for detected 26 localities in Table 1. In the results section, the names are given in alphabetical order within the families, and the number of examined specimens and collecting localities that are written according to the codes in Table 1, are provided for each species. In addition, the species are also assessed by their local, rare or widespread distribution in the studied area, according to the evaluations based on the number of captured samples and observations during the field studies.

Table 1. Coordinates, altitudes and studied dates of the localities

\begin{tabular}{|c|c|c|c|c|}
\hline Code & Locality & Coordinates & Altitude & Dates of Study \\
\hline 1 & Bekhal & $36^{\circ} 36^{\prime} 17^{\prime \prime N} 44^{\circ} 31^{\prime} 41^{\prime \prime E}$ & $760 \mathrm{~m}$ & $10 / 5 / 2017$ \\
\hline 2 & Choman & $36^{\circ} 34^{\prime} 49^{\prime \prime N} 44^{\circ} 51^{\prime} 37^{\prime \prime} \mathrm{E}$ & $1060 \mathrm{~m}$ & $12 / 7 / 2017$ \\
\hline 3 & Dlope & $36^{\circ} 11^{\prime} 26^{\prime \prime N} 44^{\circ} 07^{\prime} 03^{\prime \prime E}$ & $750 \mathrm{~m}$ & 08/5/2017 \\
\hline 4 & Dole Sakran & $36^{\circ} 54^{\prime} 58^{\prime \prime N} 44^{\circ} 44^{\prime} 53^{\prime \prime E}$ & $1325 \mathrm{~m}$ & 09/8/2017 \\
\hline 5 & Elnjakh & $35^{\circ} 58^{\prime} 12^{\prime \prime N} 44^{\circ} 30^{\prime} 50^{\prime \prime E}$ & $470 \mathrm{~m}$ & $01 / 5 / 2017$ \\
\hline 6 & Faqayan & $36^{\circ} 15^{\prime} 52^{\prime \prime N} 44^{\circ} 20^{\prime} 15^{\prime \prime E}$ & $860 \mathrm{~m}$ & $07 / 5 / 2017$ \\
\hline 7 & Gazna & $36^{\circ} 14^{\prime} 20^{\prime \prime N} 43^{\circ} 56^{\prime} 01^{\prime \prime E}$ & $390 \mathrm{~m}$ & 09/5/2017 \\
\hline 8 & Haebat Sultan & $36^{\circ} 22^{\prime} 25^{\prime \prime N} 44^{\circ} 10^{\prime} 26^{\prime \prime E}$ & $835 \mathrm{~m}$ & $05 / 5 / 2017$ \\
\hline 9 & Hawawan & $36^{\circ} 03^{\prime} 32^{\prime \prime} \mathrm{N} 44^{\circ} 42^{\prime} 26^{\prime \prime} \mathrm{E}$ & $700 \mathrm{~m}$ & $04 / 4 / 2017$ \\
\hline 10 & Heran & $36^{\circ} 17^{\prime} 04^{\prime \prime N} 44^{\circ} 29^{\prime} 33^{\prime \prime E}$ & $850 \mathrm{~m}$ & $27 / 4 / 2017$ \\
\hline 11 & Jale & 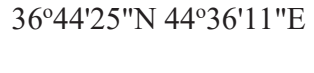 & $740 \mathrm{~m}$ & $\begin{array}{l}25 / 4 / 2016,27 / 3 / 2017,20 / 5 / 2017, \\
18 / 6 / 2017,06 / 7 / 2017\end{array}$ \\
\hline 12 & Kane Qara & $36^{\circ} 04^{\prime} 29^{\prime \prime} \mathrm{N} 44^{\circ} 39^{\prime} 15^{\prime \prime} \mathrm{E}$ & $555 \mathrm{~m}$ & $\begin{array}{l}\text { 04/5/2016, 14/3/2017, 22/4/2017, } \\
08 / 7 / 2017\end{array}$ \\
\hline 13 & Khabat & $36^{\circ} 14^{\prime} 40^{\prime \prime} \mathrm{N} 43^{\circ} 43^{\prime} 09^{\prime \prime} \mathrm{E}$ & $400 \mathrm{~m}$ & $02 / 8 / 2017$ \\
\hline 14 & Khalefan & $36^{\circ} 36^{\prime} 19^{\prime \prime N} 44^{\circ} 24^{\prime} 22^{\prime \prime E}$ & $660 \mathrm{~m}$ & $11 / 7 / 2017$ \\
\hline 15 & Kosar & $36^{\circ} 06^{\prime} 30^{\prime \prime N} 44^{\circ} 40^{\prime} 09^{\prime \prime} \mathrm{E}$ & $900 \mathrm{~m}$ & $13 / 5 / 2017$ \\
\hline 16 & Mala Omer & 36056'56"N 44ㄴ'2'26"E & $570 \mathrm{~m}$ & $05 / 4 / 2017$ \\
\hline 17 & Mawaran & $36^{\circ} 27^{\prime} 52^{\prime \prime N} 44^{\circ} 15^{\prime} 20^{\prime \prime} \mathrm{E}$ & $760 \mathrm{~m}$ & 29/4/2017 \\
\hline 18 & Meer Said & $36^{\circ} 24^{\prime} 44^{\prime \prime N} 44^{\circ} 30^{\prime} 51^{\prime \prime E}$ & $840 \mathrm{~m}$ & 04/5/2017, 05/7/2017 \\
\hline 19 & Sami Abdulrahman Park & 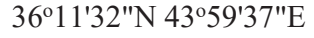 & $420 \mathrm{~m}$ & $13 / 7 / 2017$ \\
\hline 20 & Shaqlawa & 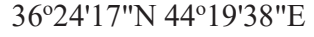 & $800 \mathrm{~m}$ & $10 / 7 / 2017$ \\
\hline 21 & Shekharwan & $36^{\circ} 04^{\prime} 31^{\prime \prime N} 44^{\circ} 32^{\prime} 39^{\prime \prime} \mathrm{E}$ & $630 \mathrm{~m}$ & 08/4/2017 \\
\hline 22 & Shewe Samal & $36^{\circ} 06^{\prime} 02^{\prime \prime N} 44^{\circ} 31^{\prime} 34^{\prime \prime E}$ & $770 \mathrm{~m}$ & $18 / 4 / 2017$ \\
\hline 23 & Sktan & $36^{\circ} 13^{\prime} 57^{\prime \prime N} 44^{\circ} 36^{\prime} 46^{\prime \prime E}$ & $695 \mathrm{~m}$ & $26 / 4 / 2017$ \\
\hline 24 & Soran & $36^{\circ} 39^{\prime} 28^{\prime \prime N} 44^{\circ} 30^{\prime} 54^{\prime \prime E}$ & $550 \mathrm{~m}$ & $12 / 5 / 2017$ \\
\hline 25 & Taq Taq & $35^{\circ} 54^{\prime} 45^{\prime \prime N} 44^{\circ} 36^{\prime} 39^{\prime \prime} \mathrm{E}$ & $400 \mathrm{~m}$ & $06 / 4 / 2017$ \\
\hline 26 & Ttopco & 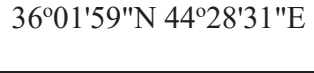 & $525 \mathrm{~m}$ & $\begin{array}{l}\text { 19/4/2017, 21/4/2017, 22/4/2017, } \\
15 / 6 / 2017\end{array}$ \\
\hline
\end{tabular}

\section{Results}

\subsection{Superfamily: Papilionoidea}

\subsubsection{Family: Papilionidae}

Archon apollinaris (Staudinger, [1892])

Material: 9 specimens. Localities: 11, 17, 12, 25.

Distribution: Local.

Iphiclides podalirius (Linnaeus, 1758)
Material: 19 specimens. Localities: 4. Distribution: Local.

Papilio machaon Linnaeus, 1758

Material: 2 specimens. Localities: 26. Distribution: Rare.

Princeps demoleus Linnaeus, 1758

Material: 4 specimens. Localities: 12. Distribution: Rare. 
Zerynthia deyrollei (Oberthür, 1869)

Material: 104 specimens. Localities: 1, 5, 10, 11, 12, 16, 17, 23, 25. Distribution: Widespread.

\subsubsection{Family: Pieridae}

Anthocharis cardamines (Linnaeus, 1758)

Material: 32 specimens. Localities: 11, 17, 25.

Distribution: Widespread. Aporia crataegi (Linnaeus, 1758)

Material: 82 specimens. Localities: 1, 6, 15, 18, 24.

Distribution: Widespread.

Colias crocea (Fourcroy, 1785)

Material: 28 specimens. Localities: 1, 4, 6, 11, 12,

19, 20, 25. Distribution: Widespread.

Euchloe belemia (Esper, [1800])

Material: 52 specimens. Localities: 1, 3, 5, 7, 8, 9,

12, 18, 21, 22, 23, 25, 26. Distribution: Widespread.

Pieris brassicae (Linnaeus, 1758)

Material: 71 specimens. Localities: 1, 5, 6, 11, 12,

14, 16, 18. Distribution: Widespread.

Pieris ergane (Geyer, [1828])

Material: 13 specimens. Localities:

Distribution: Local.

Pieris pseudorapae Verity, 1908

Material: 10 specimens. Localities: 3, 12.

Distribution: Local.

Pieris rapae (Linnaeus, 1758)

Material: 112 specimens. Localities: 1, 2, 3, 4, 5, 7,

8, 11, 12, 14, 19, 20, 24. Distribution: Widespread. Pontia daplidice (Linnaeus, 1758)

Material: 10 specimens. Localities: 11, 12, 20.

Distribution: Local.

Pontia edusa (Fabricius, 1777)

Material: 15 specimens. Localities: 1, 3, 4, 8, 16.

Distribution: Widespread.

Zegris eupheme (Esper, [1804])

Material: 6 specimens. Localities: 26. Distribution:

Rare.

\subsubsection{Family: Nymphalidae} 1775)

Argynnis pandora ([Denis \& Schiffermüller],

Material: 6 specimens. Localities: 4. Distribution: Local.

Limenitis reducta Staudinger, 1901

Material: 8 specimens. Localities: 4. Distribution:

Local.

Melitaea arduinna (Fabricius, 1787)

Material: 2 specimens. Localities: 17. Distribution:

Rare.

Melitaea fascelis (Fabricius, 1787)

Material: 6 specimens. Localities: 17, 21, 25.

Distribution: Local.

Melitaea phoebe (Goeze, 1779)

Material: 5 specimens. Localities: 1, 11, 17. Distribution: Local.

Polygonia egea (Cramer, [1775])
Material: 10 specimens. Localities: 18, 24.

Distribution: Local.

Vanessa cardui (Linnaeus, 1758)

Material: 35 specimens. Localities: 1, 6, 11, 12.

Distribution: Widespread.

\subsubsection{Family: Satyridae}

Brintesia circe (Fabricius, 1775)

Material: 5 specimens. Localities: 11, 26.

Distribution: Local.

Coenonympha pamphilus (Linnaeus, 1758)

Material: 33 specimens. Localities: 10, 11, 22, 23.

Distribution: Widespread.

Coenonympha saadi (Kollar, [1849])

Material: 22 specimens. Localities: 11, 18.

Distribution: Widespread.

Hipparchia fatua (Freyer, 1844)

Material: 6 specimens. Localities: 11. Distribution:

Rare.

Hyponephele lupina (Costa, [1836])

Material: 6 specimens. Localities: 4. Distribution:

Rare.

Hyponephele wagneri (Herrich-Schäffer, [1846])

Material: 1 specimen. Localities: 11. Distribution:

Rare.

Kirinia roxelana (Cramer, [1777])

Material: 2 specimens. Localities: 11. Distribution: Rare.

Lasiommata megera (Linnaeus, 1767)

Material: 29 specimens. Localities: 1, 4, 11, 18, 21, 22, 23, 25, 26. Distribution: Widespread.

Maniola jurtina (Linnaeus, 1758)

Material: 6 specimens. Localities: 4. Distribution: Rare.

Maniola telmessia (Zeller, 1847)

Material: 55 specimens. Localities: 1, 11, 24.

Distribution: Widespread.

Melanargia hylata (Klug, 1832)

Material: 38 specimens. Localities: 6, 11, 15.

Distribution: Widespread.

Pararge aegeria (Linnaeus, 1758)

Material: 1 specimen. Localities: 14. Distribution:

Rare.

Pseudochazara telephassa (Geyer, [1827])

(confused with $P$. anthelea (Hübner, [1824]) in the thesis) Material: 1 specimen. Localities: 7. Distribution: Rare.

\subsubsection{Family: Lycaenidae}

Chilades galba (Lederer, 1855)

Material: 38 specimens. Localities: 2, 11, 12, 13,

19. Distribution: Widespread.

Chilades trochylus (Freyer, [1843])

Material: 4 specimens. Localities: 4. Distribution:

Rare.

Glaucopsyche alexis (Poda, 1761) 
Material: 116 specimens. Localities: 1, 6, 10, 11, 17, 22, 23, 25, 26. Distribution: Widespread.

Lampides boeticus (Linnaeus, 1767)

Material: 3 specimens. Localities: 11, 12, 18.

Distribution: Local.

Lycaena phlaeas (Linnaeus, 1761)

Material: 2 specimens. Localities: 11. Distribution:

Rare.

Lycaena tityrus (Poda, 1761)

Material: 13 specimens. Localities: 2, 12, 13, 20.

Distribution: Local.

Plebejus sephirus (Frivaldszky,1835)

(confused with Polyommatus icarus (Rottemburg,

1775) in the thesis) Material: 9 specimens.

Localities: 4. Distribution: Rare.

Polyommatus agestis ([Denis \&

Schiffermüller], 1775)

Material: 9 specimens. Localities: 2, 3, 11, 25.

Distribution: Local.

Polyommatus alcestis (Zerny,1932)

Material: 15 specimens. Localities: 4. Distribution:

Rare.

Pseudophilotes vicrama (Moore, 1865)

Material: 4 specimens. Localities: 4. Distribution:

Rare.

Satyrium marcidum (Riley, 1921)

Material: 50 specimens. Localities: 1, 24.

Distribution: Local.

Tarucus balkanicus (Freyer, [1843])

Material: 7 specimens. Localities: 16, 4. Distribution: Local.

Tomares callimachus (Eversmann, 1848)

Material: 2 specimens. Localities: 9. Distribution: Rare.

Zizeeria karsandra (Moore, 1865)

Material: 9 specimens. Localities: 12, 13. Distribution: Local.

\subsection{Superfamily: Hesperioidea}

\subsubsection{Family: Hesperiidae}

Carcharodus alceae (Esper, [1780])

Material: 14 specimens. Localities: 12, 13, 19, 20.

Distribution: Local.

Erynnis tages (Linnaeus, 1758)

Material: 4 specimens. Localities: 4. Distribution: Rare.

Gegenes pumilio (Hoffmannsegg, 1804)

Material: 6 specimens. Localities: 12, 13.

Distribution: Rare.

Spialia orbifer (Hübner, [1823])

Material: 10 specimens. Localities: 6, 10, 23. Distribution: Local.

Thymelicus sylvestris (Poda, 1761)

Material: 80 specimens. Localities: 1, 3, 8, 11, 15, 24. Distribution: Widespread.

\section{Discussion}

Unfortunately, there was no research on butterflies of Erbil until now. Although, Wiltshire (1937a, 1937b, 1939a, 1939b, 1957) has given some butterfly species from northern Iraq in his papers, in which the exact location is problematic, as there is no location information for each species. Comprehensive or/and current studies on butterflies in Iraq was known by these studies: Wiltshire (1957) was recorded 136 butterflies (117 Papilionoidea, 19 Hesperioidea) from north region of Iraq, Koçak and Kemal (2001) mentioned 145 species of butterflies (123 Papilionoidea, 22 Hesperioidea), and in the study "a synonymical, and distributional checklist of the Papilionoidea and Hesperioidea of East Mediterranean countries, including Turkey (Lepidoptera)" (Kemal and Koçak, 2011) the number has risen to 151 butterflies (129 Papilionoidea, 22 Hesperioidea). Tshikolovets et al. (2014) were presented 413 species from Iran and Iraq. Also, Lahony et al. (2013) were studied flora and fauna of north-east of Iraq in general and reported 7 species of butterfly.

With this study, 55 butterfly species (50 Papilionoidea, 5 Hesperioidea) are recorded as new to the fauna of Erbil Province. These species belongs to six families: Lycaenidae (14), Satyridae (13), Pieridae (11), Nymphalidae (7), Papilionidae (5) and Hesperiidae (5). Due to the security conditions in the region, field studies have been insufficient, and the number of species in the families were not at the desired level. This number is expected to increase with future researches.

The majority of the collected 55 species are composed of rare and local individuals (Table 2). Particularly, Papilio machaon Linnaeus, 1758 , Melitaea arduinna (Fabricius, 1787), Hyponephele wagneri (Herrich-Schäffer, [1846]), Pararge aegeria (Linnaeus, 1758), Pseudochazara telephassa (Geyer, [1827]), Lycaena phlaeas (Linnaeus, 1761) and Tomares callimachus (Eversmann, 1848) species are found very rare in butterfly fauna of Erbil.

Table 2. Distribution of the species in the region according to the families

\begin{tabular}{lcccc}
\hline & Rare & Local & Widespread & Total \\
\hline Papilionidae & 2 & 2 & 1 & 5 \\
Pieridae & 1 & 3 & 7 & 11 \\
Nymphalidae & 1 & 5 & 1 & 7 \\
Satyridae & 7 & 1 & 5 & 13 \\
Lycaenidae & 6 & 6 & 2 & 14 \\
Hesperiidae & 2 & 2 & 1 & 5 \\
\hline Total & 19 & 19 & 17 & 55
\end{tabular}




\section{References}

Abusarhan, M.A., Handal, E.N., Ghattas, M.M., Qumsiyeh, M.B., Amr, Z.S., 2016. Some records of butterflies (Lepidoptera) from the palestinian territories. Jordan Journal of Biological Sciences, 9(1): 11-23.

Akın, K., 2008. Ceylanpınar (Şanlıurfa) Papilionoidea ve Hesperioidea (Lepidoptera) faunas1 ve ekolojisi üzerine araştırmalar. Yüksek lisans tezi, Van Yüzüncü Y1l Üniversitesi, Van.

Anonymous, 2017. USAID Primary Healthcare Project in Iraq. $\quad \mathrm{http} / / /$ phciraq.org/content/erbil-provinceprimary-health-care-phc-centers (Accessed: 15.12.2017)

Aussem, B., 1980. Satyriden-studie I. zur kenntnis der androkonienfelder von Pseudochazara telephassa (Geyer, 1827) und Pseudochazara anthelea (Hübner, 1824). Entomofauna, 1(17): 354-356.

Back, W., 2012. Phaenotypische und genotypische abgrenzung der arten und unterarten der gattung Zegris boisduval, 1836 (Lepidoptera, Pieridae). Atalanta, 43(1-2): 77-86.

Benyamini, D., Bruschini, C., Serra, G., John. E., 2007. First confirmed records of Papilio demoleus (Linnaeus, 1758) in Syria, with comments on the species appearance in Turkey and the Dominican Republic. News of the Israel Lepidopterists Society, 24(2): 4-11.

Coenen, F., Olivier, A., 1989. Au sujet du statut taxonomique d'Euchloe belemia moslemi Seyer, 1988 (Lepidoptera, Pieridae). Phegea, 17(1): 19-25.

Freina, J.J., De Naderi, A.R., 2003. Beschreibung einer neuen Unterart von Archon apollinaris (Staudinger, [1892]) aus dem südwestlichen Zentral-Zagros, bostanchii subspec.nov. mit ergänzenden angaben zur gesamtverbreitung der art (Papilionidae, Parnassiini). Atalanta, 34(3-4): 429-434.

Geiger, H., Descimon, H., Scholl, A., 1988. Evidence for speciation within nominal Pontia daplidice (Linnaeus, 1758) in southern Europe (Lepidoptera, Pieridae). Nota lepidopterologica, 11(1): 7-20.

Graves, P.P., 1925. The Rhopalocera and Grypocera of Palestine and Transjordania. Transactions of the Entomological Society of London, 73(1-2): 17-125.

Hardy, P.B., 1995. Butterflies in Jordan, Syria and Lebanon. Entomologists Record, 107: 107-116.

Hemming, A.F., 1932. The butterflies of Transjordan. Transactions of the Entomological Society of London, 80(2): 269-300.

Hesselbarth, G., Oorschot, H.V., Wagener, S., 1995. Die Tagfalter der Türkei. Verlag Goecke \& Evers, Bochum.

Higgins, L.G., 1958. Butterflies in Kurdistan. Entomologists, 91: 38-45.

Kemal, M., Koçak, A.Ö., 2011. Asynonymical, and disributional checklist of the Papilionoidea and Hespeirioidea of East Mediterranean countries, Including Turkey (Lepidoptera). Priamus (Supplement), 25: 1-162.
Koçak, A.Ö., Kemal, M., 2001. Studies on the geography of the Lepidoptera 3. zoogeographical remarks on the butterflies of Iraq (Lepidoptera, Papilionoidea, Hesperioidea). Priamus 10(3-4): 164-173 (in Uighur language).

Koçak, A.Ö., Kemal, M., 2007. Synonymical and distributional list of the species of Şırnak province (South East Turkey) (Lepidoptera). Centre for Entomological Studies Ankara, 127:1-8.

Koçak, A.Ö., Kemal, M., 2014. Lepidoptera of Iran based upon the info-system of the cesa. Priamus (Supplement), 31: 1-487.

Koçak, A.Ö., Kemal, M., 2015a. Annotated list of the Lepidoptera of Hakkari province (SE Turkey). Cesa News, 116: 1-146.

Koçak, A.Ö., Kemal, M., 2015b. List of the Lepidoptera of Mardin province (SE Turkey). Cesa News, 103: 27-37.

Koçak, A.Ö., Kemal, M., Kayci, L., 2011. List of the Species of the Lepidoptera of Van province (East Turkey). Centre for Entomological Studies, Priamus (Supplement), 23: 1-43.

Lahony, A.S.R., Mohammad, M.K., Ali, H.H., AlMoussawi, A.A., Abd Al-Rasul, M.S., 2013. Fauna and flora of Hawraman Mountain (Part One): Hawraman lowest one, Kurdistan province North East of Iraq. Bulletin of the Iraq Natural History Museum, 12(4): 7-34.

Larsen, T.B., 1974. Butterflies of Lebanon. Beirut, National Council for Scientific Research, Lebanon.

Seven, E., 2010. Studies on the fauna and ecology of Papilionoidea and Hesperioidea (Lepidoptera) in Sirvan district (Siirt Prov., SE Turkey). Priamus (Supplement), 20: 1-118 (in Turkish).

Seyer, H., 1988. Euchloe belemia moslemi ssp.n. de l'Iraq (Lepidoptera, Pieridae). Linneana Belgica, 11: 329332.

Tshikolovets, V., Naderi, A., Eckweiler, W., 2014. The Butterflies of Iran and Iraq. Tshikolovets Publications, Pardubice.

Wiltshire, E.P., 1937a. Autumnal Lepidoptera in Kurdistan. Preliminary notes on some excursions in the Rowanduz Chai Valley, Iraq. The entomologist's record and journal of variation, 49: 91-94.

Wiltshire, E.P., 1937b. Autumnal Lepidoptera in Kurdistan. Preliminary notes on some excursions in the Rowanduz Chai Valley, Iraq. The entomologist's record and journal of variation, 49: 107-109.

Wiltshire, E.P., 1939a. More notes on Kurdish Lepidoptera, with description of a new race and comparisons with Luristan and Alvand, Iran. The Entomologist's Record and Journal of Variation, 51: 97-103.

Wiltshire, E.P., 1939b. More notes on Kurdish Lepidoptera, with description of a new race and comparisons with Luristan and Alvand, Iran. The Entomologist's Record and Journal of Variation, 51: 133-138.

Wiltshire, E.P., 1957. The Lepidoptera of Iraq. The Minister of Agriculture Goverment of Iraq, Iraq. 